\title{
Magnetoresistance in Two-Component Systems
}

\author{
P. S. Alekseev, ${ }^{1}$ A. P. Dmitriev, ${ }^{1}$ I. V. Gornyi ${ }^{2,1,3}$ V. Yu. Kachorovskii, ${ }^{1}$ B. N. Narozhny, ${ }^{3,4}$ M. Schütt, ${ }^{5}$ and M. Titov ${ }^{6, *}$ \\ ${ }^{1}$ A.F. Ioffe Physico-Technical Institute, 194021 St. Petersburg, Russia \\ ${ }^{2}$ Institut für Nanotechnologie, Karlsruhe Institute of Technology, 76021 Karlsruhe, Germany \\ ${ }^{3}$ Institut für Theorie der Kondensierten Materie, Karlsruhe Institute of Technology, 76128 Karlsruhe, Germany \\ ${ }^{4}$ National Research Nuclear University MEPhI (Moscow Engineering Physics Institute), 115409 Moscow, Russia \\ ${ }^{5}$ School of Physics and Astronomy, University of Minnesota, Minneapolis, Minnesota 55455, USA \\ ${ }^{6}$ Radboud University, Institute for Molecules and Materials, NL-6525 AJ Nijmegen, Netherlands \\ (Received 18 October 2014; revised manuscript received 7 February 2015; published 14 April 2015)
}

\begin{abstract}
Two-component systems with equal concentrations of electrons and holes exhibit nonsaturating, linear magnetoresistance in classically strong magnetic fields. The effect is predicted to occur in finite-size samples at charge neutrality due to recombination. The phenomenon originates in the excess quasiparticle density developing near the edges of the sample due to the compensated Hall effect. The size of the boundary region is of the order of the electron-hole recombination length that is inversely proportional to the magnetic field. In narrow samples and at strong enough magnetic fields, the boundary region dominates over the bulk leading to linear magnetoresistance. Our results are relevant for two-and three-dimensional semimetals and narrow band semiconductors including most of the topological insulators.
\end{abstract}

DOI: 10.1103/PhysRevLett.114.156601

PACS numbers: 72.20.My, 71.28.+d

Growing interest in narrow band semiconductors such as topological insulators and semimetals (e.g., graphene) continues to stimulate intense experimental research. An increasing number of these studies report observations of large linear magnetoresistance (MR), which often shows no sign of saturation in classically strong magnetic fields even at room temperatures [1-8].

The story of linear MR in nonmagnetic compounds, notably in compensated semimetals $[9,10]$, can be traced back to the work by Kapitza in 1928 on bismuth [11]. The topic has received revived attention after the discovery of huge linear MR in bismuth films [12,13], as well as in AgSe and AgTe compounds [14-18], which are narrow band semiconductors [19]. A linear increase of resistance by 3 orders of magnitude has been seen in these experiments in a wide range of temperatures. The term "titanic magnetoresistance" was coined very recently in Refs. [20-23], where both linear and nonlinear change of resistance in CdAs, WTe, and NbSb has been observed.

Most of the conventional transport theories predict either absent or parabolic MR [24]. Classical linear MR has been predicted by Azbel for a three-dimensional metallic slab with smooth boundaries $[25,26]$. Quantum linear MR has been found by Abrikosov [27] for Dirac fermions in compensated semimetals in the extreme limit, $\omega_{c} \gg T$ (only one Landau level is filled), which is not reached in most measurements (here, $\omega_{c}$ is the cyclotron frequency, $T$ is the temperature, and $\hbar=k_{B}=1$ ). In weak magnetic fields the linear behavior may also be associated with quantum interaction corrections $[28,29]$ or with the classical "corridor" effect [30]. Certain specific features of excitation spectra and models of disorder may also lead to linear MR [25,31-35]. Experimentally, linear MR has been observed in a wide variety of $2 \mathrm{D}$ and $3 \mathrm{D}$ materials [1-9,11-18,36-42] and in a broad range of magnetic fields, suggesting that a more general mechanism is behind the observed behavior.

A classical approach to magnetotransport that is independent of the details of excitation spectra has been put forward by Parish and Littlewood on the basis of a classical random-resistor model [43]. This theory was argued to describe a strongly inhomogeneous (or granular) material, such as AgSe. It is unclear, however, whether the same approach can be used to capture the physics of clean, homogeneous systems, such as the high-quality BiSb nanosheets [4] and homogeneous monocrystalline $\mathrm{HgTe} / \mathrm{CdTe}$ samples [6,8]. Moreover, several experiments $[4,17,18]$ indicate that the necessary condition for the nonsaturating, linear MR is the presence of two types of charge carriers, e.g., electrons and holes, in nearly equal concentrations.

In this Letter, we propose a classical mechanism for linear MR in finite-size samples near charge neutrality (charge compensation). Our approach is based on the kinetic theory for a two-component system. The dominant contribution to the effect originates in the narrow regions near the sample edges; see Fig. 1.

The conventional Drude theory predicts that the longitudinal resistivity of a two-component system $[9,10]$ depends on the applied magnetic field (in contrast to the simplest one-component case [24,26,44]):

$$
\rho_{x x}=\frac{\rho}{e \mu} \frac{1+(\mu B)^{2}}{\rho^{2}+n^{2}(\mu B)^{2}}
$$

Here, $B$ is the magnetic field, $\mu$ is the mobility (for simplicity, the mobility is taken to be the same for electrons 


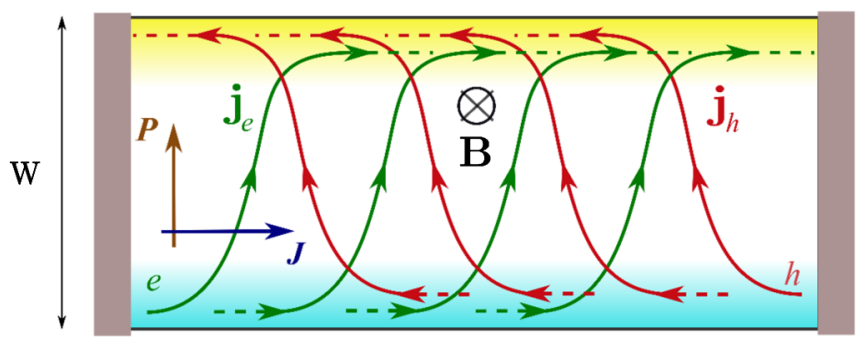

FIG. 1 (color online). Electron (green) and hole (red) trajectories in an electron-hole symmetric setup at charge neutrality. The bulk of the sample exhibits large geometric MR as a consequence of the compensated Hall effect: electron and hole trajectories are tilted but the Hall voltage is absent. Lateral quasiparticle flow $\boldsymbol{P}$ results in excess quasiparticle density near the sample edges, where recombination processes due to electron-phonon interaction lead to linear MR.

and holes), $e$ is the absolute value of the electron charge, $\rho=n_{e}+n_{h}$ is the quasiparticle density, and $n=n_{e}-n_{h}$ is the charge density per unit charge with $n_{e(h)}$ standing for the corresponding electron (hole) densities. Equation (1) predicts vanishing MR far from the charge neutrality, $n=\rho=n_{e}$, and a nonsaturating, quadratic MR at charge neutrality, $n=0$, where the Hall effect is compensated: $\sigma_{x y}=\rho_{x y}=0$.

At charge neutrality, the above result corresponds to a constant quasiparticle flow, $\boldsymbol{P}=\boldsymbol{j}_{e}+\boldsymbol{j}_{h}$, which is orthogonal to the electric current, $\boldsymbol{J}=-e \boldsymbol{j}=-e\left(\boldsymbol{j}_{e}-\boldsymbol{j}_{h}\right.$ ) (here, $\boldsymbol{j}_{e}$ and $\boldsymbol{j}_{h}$ are the electron and hole current densities), due to the classical Hall effect. The lateral quasiparticle flow $\boldsymbol{P}$ cannot be affected by the Hall voltage since the latter is not formed at charge neutrality. On the other hand, the quasiparticle current must vanish at the sample boundaries. Thus, the result of Eq. (1) is, strictly speaking, incompatible with finite-size geometry.

Here we demonstrate that boundary effects may significantly modify Eq. (1), leading to nonsaturating, linear MR near the charge neutrality point when the sample width is comparable with the electron-hole recombination length $\ell_{0}$. The latter may vary from hundreds of nanometers to centimeters depending on material properties and temperature. The role of the quasiparticle recombination in "anisotropic size effects" in narrow band semiconductors and semimetals was stressed long ago by Rashba and co-workers [45]. A related phenomenon was suggested recently to be responsible for a negative Coulomb drag in graphene [46].

To develop intuition for the boundary effect, let us consider a rectangular 2D sample of length $L$ and width $W$; see Fig. 1 . The same arguments apply to a 3D slab in a magnetic field parallel to its surfaces. For simplicity, we assume an electron-hole symmetric system at charge neutrality, where the electric current $\boldsymbol{J}$ is injected in the $x$ direction. Since the classical Hall effect for electrons compensates that for holes, the electrostatic potential in the sample remains flat and the charge density is zero everywhere, $n=0$. The distribution of electron and hole currents $\boldsymbol{j}_{e, h}$, however, is nontrivial: it is essentially different in the bulk of the sample and in the boundary regions, see Fig. 1 (resembling the "static skin effect" [25]). In the bulk, the transverse quasiparticle current $\boldsymbol{P}=\boldsymbol{j}_{e}+\boldsymbol{j}_{h}$ leads to geometric MR, $R_{\text {bulk }}=(L / W)\left(1+\mu^{2} B^{2}\right) /(e \rho \mu)$ [see Eq. (1) for $n=0$ ]. In single-component systems, such a geometric effect is absent due to the presence of Hall voltage, unless the Corbino geometry is used or the sample is specifically prepared to be short and wide, i.e., for $W \gg L[9,47]$.

The bulk current $\boldsymbol{P}$ leads to a formation of excess quasiparticle density near the sample edges at $y=$ $\pm W / 2$ (as shown in Fig. 1) that has to be relaxed by electron-hole recombination, e.g., due to electron-phonon scattering. This yields variation of the quasiparticle density over the distance $\ell_{R}=\ell_{0} / \sqrt{1+\mu^{2} B^{2}}$ from the boundary, where $\ell_{0}=2 \sqrt{D \tau_{R}}$ depends on the diffusion coefficient $D$ and the recombination time $\tau_{R}$. As $B$ increases, the recombination length $\ell_{R}$ gets shorter because of multiple cyclotron returns of electrons and holes to each other.

In the boundary regions of the size of the recombination length $\ell_{R}$, the electron and hole currents are directed essentially along the $x$ axis. Thus, the edge contribution to the overall resistance lacks the geometric enhancement and at charge neutrality is given by $(B=|\boldsymbol{B}|)$

$$
R_{\text {edge }}=L /\left(\ell_{R} e \mu \rho\right) \stackrel{\mu B \gg 1}{\longrightarrow} L B /\left(\ell_{0} e \rho\right) .
$$

The total sheet resistance $R_{\square}$ for $W \gg \ell_{R}$ is estimated by regarding the edge and the bulk as parallel resistors:

$$
R_{\square}^{-1}=(L / W)\left(R_{\text {bulk }}^{-1}+R_{\text {edge }}^{-1}\right) .
$$

This yields $R_{\square}^{-1}=e \rho \mu\left[1 /(\mu B)^{2}+\ell_{0} /(W \mu B)\right]$, where we assumed $\mu B \gg 1$. Thus, for sufficiently strong fields, the MR at charge neutrality is linear in the field, namely,

$$
R_{\square}=W B /\left(e \rho \ell_{0}\right), \quad \ell_{0} /(\mu B) \ll W \ll \mu B \ell_{0} .
$$

Remarkably, within the semiclassical Drude model any two-component neutral liquid with recombination is characterized by linear MR as $B \rightarrow \infty$.

Upon deviation from charge neutrality, the geometric resistance in the bulk of the sample disappears due to formation of the Hall voltage. From Eq. (1), for $\mu B \gg 1$ one finds $R_{\text {bulk }}^{-1}=(W / L) e \rho \mu\left[1 /(\mu B)^{2}+n^{2} / \rho^{2}\right]$, yielding

$$
R_{\square}^{-1}=e \rho \mu\left[1 /(\mu B)^{2}+n^{2} / \rho^{2}+\ell_{0} /(W \mu B)\right] .
$$

Thus, the linear regime of Eq. (3) holds in strong fields as long as $n / \rho \ll \sqrt{\ell_{0} / W \mu B}$.

In the remainder of this Letter we use the standard equations of macroscopic electrodynamics to show that our result (3) is generic for compensated two-component 
systems. Within linear response, the electric current is proportional to the electric field $\boldsymbol{E}$ and the gradient of the carrier concentration $[48,49]$ (here, $\nu$ is the thermodynamic density of states, $q= \pm e$ is the carrier charge)

$$
J_{i}=\sigma_{i j}\left[E_{j}+(q \nu)^{-1} \partial_{j} n\right] .
$$

Inverting the conductivity matrix, we rewrite Eq. (5) as

$$
\boldsymbol{E}+q D r_{0} \boldsymbol{\nabla} n=r_{0} \boldsymbol{J}-r_{H} \boldsymbol{J} \times \boldsymbol{e}_{z},
$$

where $r_{0}$ and $r_{H}$ are the longitudinal and Hall resistivities and $\boldsymbol{e}_{z}$ is the direction of the magnetic field. The essential feature of Eq. (6) is that $D r_{0}$ is determined only by the thermodynamic density of states and is independent of the nonquantizing magnetic field [50].

The gradient term in Eq. (6) indicates inhomogeneity of quasiparticle currents and densities. In a narrow sample of length $L$ and width $W \ll L$, this appears due to the closed boundary conditions $j_{y}(y= \pm W / 2)=0$. At charge neutrality, the electric charge remains uniform (due to the vanishing Hall effect). The charge density should be determined from a self-consistent solution of the linearresponse equations and the corresponding electrostatic problem. In 2D samples and in the limit of a strong screening by the gate, the relation simplifies as

$$
\boldsymbol{E}=E_{0} \boldsymbol{e}_{x}-(q / C)(\partial \delta n / \partial y) \boldsymbol{e}_{y} .
$$

Here $E_{0}$ is the external field, $\delta n$ is the density fluctuation, $C=\epsilon / 4 \pi d$ is the gate-to-channel capacitance per unit area, $d$ is the distance to the gate, $\epsilon$ is the dielectric constant. In $3 \mathrm{D}$ samples with thickness $d_{0}$, Eq. (7) is replaced by $d E_{y} / d y=q \delta n(y) / \epsilon d_{0}, E_{x}=E_{0}$.

If the system comprises two types of carriers (e.g., electrons and holes), which are completely independent of each other, one can apply Eq. (6) to the electrons and holes [51]. The measured electric current is then given by the sum of the electron and hole contributions. In particular, at charge neutrality the Hall current vanishes since the electrons and holes carry opposite charge.

Taking into account quasiparticle recombination processes, we find that the linear-response equations (6) can still be applied independently to the electrons and holes, but should be supplemented by the continuity equations

$$
\boldsymbol{\nabla} \cdot \boldsymbol{j}_{e, h}=-\left(\Gamma_{e} \delta n_{e}+\Gamma_{h} \delta n_{h}\right) / 2,
$$

where $\delta n_{\alpha}(\boldsymbol{r})=n_{\alpha}(\boldsymbol{r})-n_{0, \alpha}$ is the density deviation from its equilibrium value $n_{0, \alpha}$ and $\Gamma_{\alpha}$ is the electron-hole recombination rate, e.g., due to electron-phonon interaction (the index $\alpha=e, h$ refers to electrons or holes).

Further analysis is greatly simplified at charge neutrality $\left(n_{0}=0\right)$ under the assumption of electron-hole symmetry: $D_{\alpha}=D, r_{0, \alpha}=r_{0}, r_{H, e}=-r_{H, h}=r_{H}$, and $\Gamma_{\alpha}=1 / \tau_{R}$. We now rewrite Eqs. (6) and (8) as

$$
\begin{gathered}
D r_{0} \nabla \delta \rho+r_{0} \boldsymbol{P}+r_{H} \boldsymbol{j} \times \boldsymbol{e}_{z}=0, \\
r_{0} \boldsymbol{j}+2 \boldsymbol{E} / e+r_{H} \boldsymbol{P} \times \boldsymbol{e}_{z}=0, \\
\boldsymbol{\nabla} \cdot \boldsymbol{P}=-\delta \rho / \tau_{R}, \quad \boldsymbol{\nabla} \cdot \boldsymbol{j}=0,
\end{gathered}
$$

where $\delta \rho=\delta n_{e}+\delta n_{h}$ is the deviation of the quasiparticle density from its equilibrium value $\rho_{0}=n_{0, e}+n_{0, h}$. In the electron-hole symmetric system, the charge fluctuations are absent: $\delta n=0$. Thus, we find $\boldsymbol{E}=E_{0} \boldsymbol{e}_{x}$ irrespective of the electrostatic properties and dimensionality of the system.

In addition to quasiparticle recombination, electronelectron interaction leads to mutual friction between electrons and holes [10]. This effect can be taken into account by adding the frictional term $r_{e h} \boldsymbol{j}$ to Eq. (9b), effectively renormalizing $r_{0}$ in this equation. This does not lead to any qualitatively new behavior of the system and is omitted below for simplicity.

The model (9) is solved by $\boldsymbol{P}=P(y) \boldsymbol{e}_{y}, \boldsymbol{j}=j(y) \boldsymbol{e}_{x}$, $\delta \rho=\delta \rho(y)$. Excluding the variation of the quasiparticle density, we rewrite the remaining equations as

$$
\begin{gathered}
-D \tau_{R} \partial^{2} P / \partial y^{2}+P(y)-\left(r_{H} / r_{0}\right) j(y)=0, \\
j(y)=-j_{0}-\left(r_{H} / r_{0}\right) P(y),
\end{gathered}
$$

where $j_{0}=2 E_{0} /\left(e r_{0}\right)$. Excluding $j(y)$ from Eqs. (10), we obtain a linear differential equation for $P(y)$ with the boundary conditions $P( \pm W / 2)=0$, solved by

$$
P(y)=P_{B}\left[\frac{\cosh \left(2 y / \ell_{R}\right)}{\cosh \left(W / \ell_{R}\right)}-1\right], \quad P_{B}=j_{0} \frac{r_{0} r_{H}}{r_{0}^{2}+r_{H}^{2}} .
$$

Here we have introduced the electron-hole recombination length $\ell_{R}=\ell_{0} r_{0} / \sqrt{r_{0}^{2}+r_{H}^{2}}$. The profile of the quasiparticle flow $P(y)$ is illustrated in Fig. 3 (bottom) along with the corresponding quasiparticle density $\delta \rho(y)$. The result (11) and the corresponding current $j(y)$ obtained from Eq. (10b) fully agree with the qualitative distribution of quasiparticle currents shown in Fig. 1.

In 2D systems (attracting substantial experimental interest in the context of linear MR) the sheet resistance is defined as

$$
R_{\square}=E_{0} / \bar{J}, \quad \bar{J}=-\frac{e}{W} \int_{-W / 2}^{W / 2} j(y) d y .
$$

From Eqs. (10b), (11), and (12) we obtain

$$
R_{\square}^{-1}=2 F\left(W / \ell_{R}\right) / r_{0}+2\left[1-F\left(W / \ell_{R}\right)\right] r_{0} /\left(r_{0}^{2}+r_{H}^{2}\right),
$$

where $F(x)=\tanh (x) / x$. The result of Eq. (13) is plotted schematically in Fig. 2 for three different values of the ratio $W / \ell_{0}$, where $\ell_{0}$ is the zero- $B$ recombination length. 


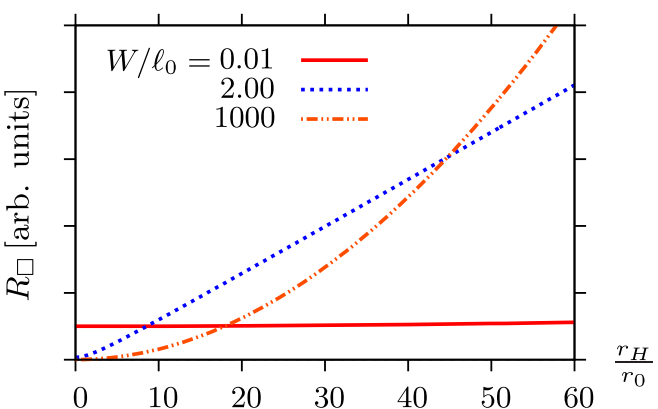

FIG. 2 (color online). Sheet resistance $R_{\square}$ at charge neutrality versus magnetic field [Eq. (13)] for three different values of the ratio $W / \ell_{0}$. The resistance is rescaled for better presentation.

Let us analyze Eq. (13) in three different regimes determined by the ratio of the sample width and recombination length. For the widest samples, we find the standard $[9,24]$ nonsaturating geometric MR, which is quadratic in the field

$$
R_{\square}=\left(r_{0}^{2}+r_{H}^{2}\right) /\left(2 r_{0}\right), \quad W \gg \ell_{R} r_{H}^{2} / r_{0}^{2} .
$$

Here the geometric enhancement is the direct consequence of the compensated Hall effect: the electron and hole trajectories are tilted, but the Hall voltage is zero.

In the narrowest samples the geometric factor is absent:

$$
R_{\square}=r_{0} / 2, \quad W \ll \ell_{R} .
$$

In this case, both electron and hole currents flow along the $x$ axis due to strong electron-hole recombination.

In classically strong magnetic fields, $r_{H} \gg r_{0}$, there exists another regime of intermediate system widths, where resistance depends linearly on the magnetic field:

$$
R_{\square}=W r_{0} /\left(2 \ell_{R}\right) \propto B, \quad \ell_{R} \ll W \ll \ell_{R} r_{H}^{2} / r_{0}^{2},
$$

where we assume the typical situation [50] where $r_{0}$ is independent of $B$ and $r_{H} \propto B$. Within the Drude model $r_{H} / r_{0}=\mu B, r_{0}=2 / e \mu \rho$, and Eq. (16) coincides with Eq. (3). If electron-hole friction $r_{e h}$ is taken into account, the result (16) acquires the extra factor $\sqrt{1+r_{e h} / r_{0}}$.

In an experimentally relevant 3D setup made of a slab of thickness $W$ subject to a magnetic field parallel to its side boundaries, our theory predicts the same results (13)-(16) for the resistivity (in particular, the linear magnetoresistivity in strong fields), up to a geometric factor. This follows directly from Eqs. (9) and the absence of induced electric field at charge neutrality.

Our macroscopic model is representative in a wide class of two-component systems in a wide range of magnetic fields and temperatures. All microscopic details (i.e., the excitation spectrum, model of disorder, etc.) are encoded in $\ell_{R}, r_{0}$, and $r_{H}$. The model (9) ignores quantum effects: we assume $T \tau_{\alpha} \gg 1$ (where $\tau_{\alpha}$ is the elastic mean-free time),
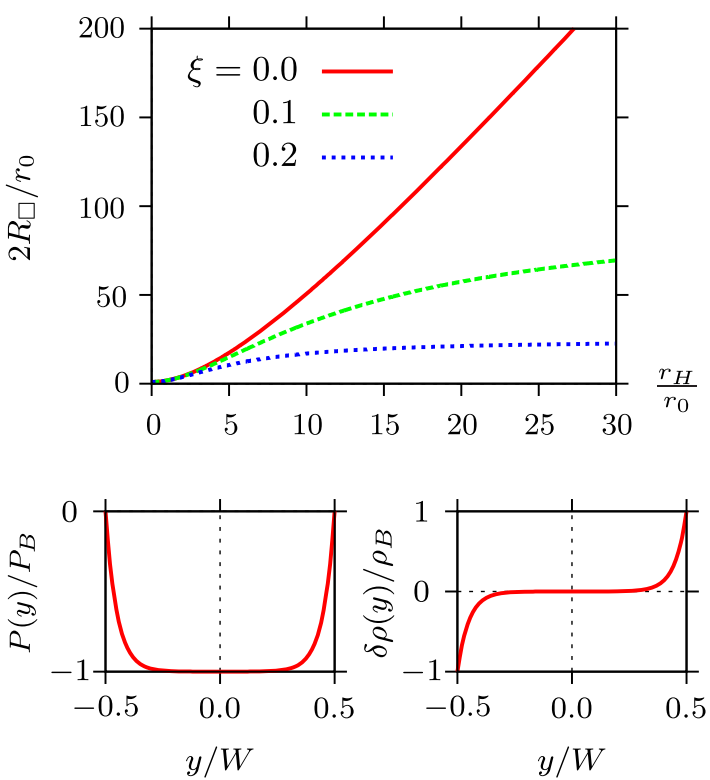

FIG. 3 (color online). Sheet resistance $R_{\square}$ (top) versus magnetic field as given by Eq. (17) for $W / \ell_{0}=10$ and different values of the parameter $\xi=n_{0} / \rho_{0}$. The bottom panels illustrate the spatial profiles of the quasiparticle current [Eq. (11)] and density [obtained from Eq. (9c)] at charge neutrality for $W / \ell_{R}=$ 10 that are measured in the units of $P_{B}$ and $\rho_{B}=2 P_{B} \tau_{R} / \ell_{R}$.

overlapping Landau levels $\omega_{\alpha} \ll T$, and, hence, the fieldindependent recombination rates $\Gamma_{\alpha}$.

In Supplemental Material [52] we present a solution away from charge neutrality and for inequivalent electron and hole bands. This solution exhibits linear MR, as in the case of equivalent bands, Eq. (16). For an electronhole-symmetric system close to charge neutrality $\left(\xi=n_{0} / \rho_{0} \ll 1\right)$ and for $r_{0} C D \ll 1$, the general result simplifies to

$$
R_{\square}=\frac{r_{0}}{2} \frac{1+\left(r_{H} / r_{0}\right)^{2}}{1+\left(r_{H} / r_{0}\right)^{2}\left[\xi^{2}+F\left(W / \ell_{R}\right)\right]} .
$$

In the limit of strong field, $r_{H} / r_{0} \gg 1$, Eq. (17) can also be anticipated from Eq. (4). The result (17) is illustrated in Fig. 3 (top panel). The MR is strongly peaked at charge neutrality (the same holds also for inequivalent electrons and holes [52]). We conclude that the simplified electronhole-symmetric model (9) captures all qualitative features of the phenomenon.

To summarize, we proposed a classical, recombinationinduced mechanism of MR in compensated semimetals and narrow band semiconductors. The universal linear-in- $B$ MR arises in finite-size samples in classically strong magnetic fields due to the interplay of bulk and edge contributions. This mechanism is expected to be relevant for the explanation of linear MR observed experimentally in various twocomponent 2D and 3D systems. Microscopic calculations based on the kinetic equation approach $[53,54]$ confirm that 
our general mechanism is responsible for linear MR in both disorder- $\left(\tau_{\alpha} \ll \tau_{e e}\right)$ and interaction-dominated $\left(\tau_{\alpha} \gg \tau_{e e}\right)$ regimes in materials with different spectra. In this way we find microscopic expressions for $\ell_{R}, r_{0}$, and $r_{H}$ in conventional 2D and 3D narrow band semiconductors (including the case when the symmetry between valence and conduction bands is violated [52]), topological insulators, bilayer graphene, and in semimetals with linear spectrum, e.g., in monolayer graphene and 3D Weyl semimetals. Our theory can be further extended to inhomogeneous samples in the spirit of Ref. [55]. In this case, the result (3) is expected to hold for arbitrary system sizes with $W$ corresponding to the typical size of macroscopic inhomogeneities.

We are grateful to V. Pudalov, S. Roumyantsev, G. Vasileva, Y. Vasilyev, H. Weber, S. Wiedmann, and U. Zeitler for helpful discussions and for providing us with unpublished experimental data. The work was supported by the Dutch Science Foundation NWO/FOM 13PR3118, the EU Network Grant No. 612624 FP7-PEOPLE-2013IRSES “InterNoM," DFG-SPP 1459, and DFG-SPP 1666, GIF, the Humboldt Foundation, the Russian Foundation of Basic Research, the Dynasty Foundation, Russian Ministry of Education and Science (Contract No. 14.Z50.31.0021), and RF President Grant No. NSh-1085.2014.2.

* Corresponding author. m.titov@science.ru.nl

[1] A. L. Friedman, J. L. Tedesco, P. M. Campbell, J. C. Culbertson, E. Aifer, F. K. Perkins, R. L. Myers-Ward, J. K. Hite, C. R. Eddy, G. G. Jernigan, and D. K. Gaskill, Nano Lett. 10, 3962 (2010).

[2] R. S. Singh, X. Wang, W. C. Ariando, and A. T. S. Wee, Appl. Phys. Lett. 101, 183105 (2012).

[3] S. X. Zhang, R. D. McDonald, A. Shekhter, Z. X. Bi, Y. Li, Q. X. Jia, and S. T. Picraux, Appl. Phys. Lett. 101, 202403 (2012).

[4] M. Veldhorst, M. Snelder, M. Hoek, C. G. Molenaar, D. P. Leusink, A. A. Golubov, H. Hilgenkamp, and A. Brinkman, Phys. Status Solidi RRL 7, 26 (2013).

[5] W. Wang, Y. Du, G. Xu, X. Zhang, E. Liu, Z. Liu, Y. Shi, J. Chen, G. Wu, and X. Zhang, Sci. Rep. 3, 2181 (2013).

[6] G. M. Gusev, E. B. Olshanetsky, Z. D. Kvon, N. N. Mikhailov, and S. A. Dvoretsky, Phys. Rev. B 87, 081311(R) (2013).

[7] B. A. Assaf, T. Cardinal, P. Wei, F. Katmis, J. S. Moodera, and D. Heiman, Appl. Phys. Lett. 102, 012102 (2013).

[8] S. Wiedmann (private communication); S. Wiedmann, A. Jost, C. Thienel, C. Brüne, P. Leubner, H. Buhmann, L. W. Molenkamp, and U. Zeitler (unpublished).

[9] H. Weiss and H. Welker, Z. Phys. 138, 322 (1954).

[10] V. F. Gantmakher and Y. B. Levinson, Zh. Eksp. Teor. Fiz. 74, 261 (1978) [Sov. Phys. JETP 47, 133 (1978)]; Carrier Scattering in Metals and Semiconductors (North-Holland, Amsterdam, 1987).

[11] P. L. Kapitza, Proc. R. Soc. A 119, 358 (1928).
[12] F. Y. Yang, K. Liu, K. Hong, D. H. Reich, P. C. Searson, and C. L. Chien, Science 284, 1335 (1999).

[13] F. Y. Yang, K. Liu, K. Hong, D. H. Reich, P. C. Searson, C. L. Chien, Y. Leprince-Wang, KuiYu-Zhang, and K. Han, Phys. Rev. B 61, 6631 (2000).

[14] R. Xu, A. Husmann, T. F. Rosenbaum, M.-L. Saboungi, J. E. Enderby, and P. B. Littlewood, Nature (London) 57, 390 (1997); M. Lee, T. F. Rosenbaum, M.-L. Saboungi, and H. S. Schnyders, Phys. Rev. Lett. 88, 066602 (2002).

[15] A. Husmann, J. B. Betts, G. S. Boebinger, A. Migliori, T. F. Rosenbaum, and M.-L. Saboungi, Nature (London) 417, 421 (2002).

[16] Y. Sun, M. B. Salamon, M. Lee, and T. F. Rosenbaum, Appl. Phys. Lett. 82, 1440 (2003).

[17] X. Zhang, Q. Z. Xue, and D. D. Zhu, Phys. Lett. A 320, 471 (2004).

[18] J. Hu and T. F. Rosenbaum, Nat. Mater. 7, 698 (2008).

[19] R. Dalven and R. Gill, Phys. Rev. B 159, 645 (1967).

[20] T. Liang, Q. Gibson, M. N. Ali, M. Liu, R. J. Cava, and N. P. Ong, Nat. Mater. 14, 280 (2015).

[21] M. N. Ali, J. Xiong, S. Flynn, Q. Gibson, L. Schoop, N. Haldolaarachchige, N. P. Ong, J. Tao, and R. J. Cava, Nature (London) 514, 205 (2014).

[22] K. Wang, D. Graf, and C. Petrovic, Sci. Rep. 4, 7328 (2014).

[23] I. Pletikosić, M. N. Ali, A. V. Fedorov, R. J. Cava, and T. Valla, Phys. Rev. Lett. 113, 216601 (2014).

[24] A. B. Pippard, Magnetoresistance in Metals (Cambridge University Press, Cambridge, England, 1989); K. Seeger, Semiconductor Physics (Springer, New York, 2002).

[25] M. Ya. Azbel, Zh. Exp. Teor. Phys. 44, 983 (1963) [Sov. Phys. JETP 17, 667 (1963)].

[26] I. M. Lifshitz, M. Ya. Azbel, and M. I. Kaganov, Electron Theory of Metals (Consultants Bureau, New York, 1973).

[27] A. A. Abrikosov, Sov. Phys. JETP 29, 746 (1969); Phys. Rev. B 58, 2788 (1998); Europhys. Lett. 49, 789 (2000).

[28] G. Zala, B. N. Narozhny, and I. L. Aleiner, Phys. Rev. B 65, 020201(R) (2001); S. A. Vitkalov, K. James, B. N. Narozhny, M. P. Sarachik, and T. M. Klapwijk, Phys. Rev. B 67, 113310 (2003).

[29] T. A. Sedrakyan and M. E. Raikh, Phys. Rev. Lett. 100, 106806 (2008).

[30] A. Dmitriev, M. Dyakonov, and R. Jullien, Phys. Rev. Lett. 89, 266804 (2002); V. V. Cheianov, A. P. Dmitriev, and V. Yu. Kachorovskii, Phys. Rev. B 70, 245307 (2004).

[31] A. G. Lebed and N. N. Bagmet, Phys. Rev. B 55, R8654 (1997).

[32] A. J. Schofield and J. R. Cooper, Phys. Rev. B 62, 10779 (2000).

[33] C. M. Wang and X. L. Lei, Phys. Rev. B 86, 035442 (2012).

[34] P. S. Alekseev, A. P. Dmitriev, I. V. Gornyi, and V. Yu. Kachorovskii, Phys. Rev. B 87, 165432 (2013).

[35] H. K. Pal and D. L. Maslov, Phys. Rev. B 88, 035403 (2013).

[36] J. Ping, I. Yudhistira, N. Ramakrishnan, S. Cho, S. Adam, and M. S. Fuhrer, Phys. Rev. Lett. 113, 047206 (2014).

[37] S. Cho and M. S. Fuhrer, Phys. Rev. B 77, 081402(R) (2008).

[38] J. Jobst, Ph.D. thesis, Friedrich-Alexander-Universität Erlangen-Nürnberg, 2012. 
[39] K. Gopinadhan, Y. J. Shin, I. Yudhistira, J. Niu, and H. Yang, Phys. Rev. B 88, 195429 (2013).

[40] Y. Zhao, P. Cadden-Zimansky, F. Ghahari, and P. Kim, Phys. Rev. Lett. 108, 106804 (2012).

[41] Yu. B. Vasilyev et al. (unpublished).

[42] F. Kisslinger, C. Ott, C. Heide, E. Kampert, B. Butz, E. Spiecker, S. Shallcross, and H. B. Weber (unpublished).

[43] M. M. Parish and P. B. Littlewood, Nature (London) 426, 162 (2003).

[44] C. Kittel, Quantum Theory of Solids (Wiley, New York, 1963).

[45] E. I. Rashba, Z. S. Gribnikov, and V. Ya. Kravchenko, Usp. Fiz. Nauk 119, 3 (1976) [Sov. Phys. Usp. 19, 361 (1976)].

[46] M. Titov, R. V. Gorbachev, B. N. Narozhny, T. Tudorovskiy, M. Schütt, P. M. Ostrovsky, I. V. Gornyi, A. D. Mirlin, M. I. Katsnelson, K. S. Novoselov, A. K. Geim, and L. A. Ponomarenko, Phys. Rev. Lett. 111, 166601 (2013).

[47] S.-J. Chang, M. Bawedin, and S. Cristoloveanu, IEEE Trans. Electron Devices 61, 1979 (2014).

[48] L. D. Landau, E. M. Lifshits, and L. P. Pitaevskii, Electrodynamics of Continuous Media (Pergamon Press, New York, 1984); E. M. Lifshits and L.P. Pitaevskii, Physical Kinetics (Pergamon Press, New York, 1972).

[49] Here we assume fast thermalization due to electron-phonon coupling. For weaker phonons, Eq. (5) also includes a temperature gradient. Within linear response, this does not affect our qualitative conclusion of the existence of linear magnetoresistance in classically strong magnetic fields.

[50] The macroscopic equation (6) can be obtained directly from the Boltzmann kinetic equation. In most cases the nonquantizing magnetic field appears in the kinetic equation in the Lorentz term only. Hence, the only $B$-dependent coefficient in Eq. (6) is $r_{H}$.

[51] Parameters $r_{0}$ and $r_{H}$ have the meaning of resistivities of individual electron and hole subsystems. Nevertheless, at charge neutrality they can be measured. The total longitudinal resistance is simply given by $r_{0} / 2$, while the parameter $r_{H}$ can be found from nonlocal transport measurements; see R. V. Gorbachev, J. C. W. Song, G. L. Yu, A. V. Kretinin, F. Withers, Y. Cao, A. Mishchenko, I. V. Grigorieva, K. S. Novoselov, L. S. Levitov, and A. K. Geim, Science 346, 448 (2014).

[52] See Supplemental Material at http://link.aps.org/ supplemental/10.1103/PhysRevLett.114.156601 for the solution of Eqs. (6-8) away from charge neutrality.

[53] B. N. Narozhny, I. V. Gornyi, M. Titov, M. Schütt, and A. D. Mirlin, Phys. Rev. B 91, 035414 (2015).

[54] P. S. Alekseev et al. (to be published).

[55] M. Knap, J. D. Sau, B. I. Halperin, and E. Demler, Phys. Rev. Lett. 113, 186801 (2014). 